OPEN ACCESS

Edited by:

Floriana Campanile,

University of Catania, Italy

Reviewed by:

Ellen Stobberingh,

National Institute Public Health,

Netherlands

Sergio Flores,

Laboratorio de Genética y

Evolución (GEVOL), Chile

${ }^{*}$ Correspondence:

Mohamed M. H. Abdelbary

mabdelbary@ukaachen.de

Specialty section:

This article was submitted to Clinical Microbiology,

a section of the journal

Frontiers in Cellular and

Infection Microbiology

Received: 25 March 2021

Accepted: 20 July 2021

Published: 06 August 2021

Citation:

Abdelbary MMH, Wilms G and Conrads G (2021) A New SpeciesSpecific Typing Method for Salivarius

Group Streptococci Based on the

Dephospho-Coenzyme A Kinase

(coaE) Gene Sequencing.

Front. Cell. Infect. Microbiol. 11:685657.

doi: 10.3389/fcimb.2021.685657

\section{A New Species-Specific Typing Method for Salivarius Group Streptococci Based on the Dephospho-Coenzyme A Kinase (coaE) Gene Sequencing}

\author{
Mohamed M. H. Abdelbary*, Gerrit Wilms and Georg Conrads
}

Division of Oral Microbiology and Immunology, Department of Operative and Preventive Dentistry and Periodontology, RWTH Aachen University Hospital, Aachen, Germany

Viridans streptococci are a group of $\alpha$-hemolytic streptococcal species. They are mainly commensals, most abundant in the mouth supporting oral health. But they also include important human pathogens such as Streptococcus pneumoniae. Identification and molecular typing of viridans group streptococci are challenging, especially for members of the salivarius group. In this study, we developed a single-locus molecular typing method that is able to differentiate among the highly phylogenetically related members of the salivarius group (S. salivarius, S. vestibularis and S. thermophilus) and might support differentiation in other groups as well. This typing approach is based on the amplification and sequence analysis of the housekeeping gene dephospho-coenzyme A kinase (coaE), a gene with unrecognized taxonomic potential to date. Here, we analysed coaE gene sequences of 154 publicly available genomes and of 30 salivarius group isolates of our own collection that together belong to 20 different gram-positive bacterial (sub) species. Our results revealed that the coaE phylogeny distinguished between streptococcal and non-streptococcal genomes and that coaE gene sequences were species-specific. In contrast to MALDI-TOF MS performance, the coaE typing was able to precisely identify the phylogenetically very closely related members of the salivarius group.

Keywords: Streptococcal typing, salivarius group, dephospho-CoA kinase gene, MALDI-TOF MS, streptococcal phylogeny

\section{INTRODUCTION}

Currently, the genus Streptococcus comprises 107 (validly published and correctly named) assigned species (http://www.bacterio.net/streptococcus.html) that diverge between commensals and pathogens to various animals and to humans. Previous studies based on 16S rRNA gene sequence analysis divided most members of the genus Streptococcus into six distinct phylogenetic groups, namely the mitis, anginosus, pyogenic, bovis, mutans, and salivarius group. The latter is composed of the oral and intestinal commensals S. vestibularis and S. salivarius while 
S. thermophilus is essential for manufacturing dairy products and thus frequently consumed in high dosages. On the one hand, S. thermophilus and - to a lower extend - S. salivarius (strains K12 and M18) are important probiotics. On the other hand, several studies have shown that $S$. salivarius and $S$. vestibularis are opportunistic pathogens and can even be associated with life threating infections such as bacteremia, endocarditis and meningitis (Corredoira et al., 2005; Shewmaker et al., 2010; Srinivasan et al., 2012), the latter occurring mainly in immunocompromised patients.

Previous studies have shown the effectiveness of matrixassisted laser desorption ionization time-of-flight mass spectrometric (MALDI-TOF MS) in identifying most species among the genus Streptococcus (Friedrichs et al., 2007; Lartigue et al., 2009). However, for MALDI-TOF MS the identification and classification of viridans streptococci, in particular those of the salivarius group, remains challenging (Murray, 2010). Furthermore, a total of 11 multilocus sequence typing (MLST) schemes were developed, mainly for pathogenic streptococcal species, such as $S$. pneumoniae, S. agalactiae, S. pyogenes and $S$. bovis/equinus complex. MLST is based on amplification and sequence analysis of seven housekeeping genes. Among the salivarius group species, only $S$. thermophilus has a wellestablished MLST scheme (Yu et al., 2015). With the introduction of high-throughput sequencing, whole-genome sequence (WGS) analysis has become a valuable typing method for bacterial species due to its high discriminatory power that is able to even differentiate between closely related bacterial isolates. But this method is still expensive and time-consuming.

In this study, we developed a single-locus based typing method that is able to identify and differentiate among the highly phylogenetically related members of the streptococcal salivarius group. This method is based on the amplification and sequence analysis of the housekeeping gene encoding dephospho-coenzyme A kinase (coaE). The coaE gene was first described in Escherichia coli and later detected in several bacterial species and in archaea (Mishra et al., 2001; Shimosaka et al., 2019). The product of dephospho-enzyme A kinase is an essential enzyme responsible for catalyzing the final step (the ATP-dependent phosphorylation of dephospho-CoA) in the coenzyme A (CoA) biosynthesis, which is a fundamental cofactor for several cellular reactions in all living organisms (Spry et al., 2008). Due to its essential role in CoA-metabolism, it was previously suggested as a drug target combating Mycobacterium tuberculosis (Ballinger et al., 2019). CoaE is informative and has a length of around 600 base pairs, which is optimal for amplification. Previous studies have revealed that bacterial, archaeal and eukaryotic CoaEs are a typical pair of analogous enzymes that are structurally and evolutionarily distinct but convergently evolve to catalyze the similar cellular reaction (Galperin et al., 1998; Omelchenko et al., 2010; Shimosaka et al., 2019). Therefore, the distinct evolutionary dynamics of the coaE gene make it suitable as genetic marker for bacterial molecular typing. In addition, we demonstrate the potential discriminatory power of $c o a E$ to distinguish among several bacterial species unrelated to the genus Streptococcus.

\section{MATERIALS AND METHODS}

\section{Primer Design and PCR Amplification}

We analyzed coaE gene sequences from 154 publicly available genomes of 28 different gram-positive bacterial species that included 134 streptococcal and 20 non-streptococcal genomes (Table S1). Four to six genomes for each of the following representative species were downloaded in fasta format from the National Center for Biotechnology Information (NCBI) database: Non-streptococci: Enterococcus faecalis ( $\mathrm{n}=5)$, E. faecium $(\mathrm{n}=5)$, Listeria monocytogenes $(\mathrm{n}=5)$, Staphylococcus aureus $(n=5)$; streptococci: $S$. agalactiae $(\mathrm{n}=5)$, S. anginosus $(\mathrm{n}=5), S$. australis $(\mathrm{n}=4), S$. constellatus $(\mathrm{n}=4), S$. cristatus $(\mathrm{n}=$ 5), S. equinus $(\mathrm{n}=5), S$. gordonii $(\mathrm{n}=5)$, S. infantis $(\mathrm{n}=5)$, $S$. intermedius $(\mathrm{n}=6), S$. mitis $(\mathrm{n}=5), S$. mutans $(\mathrm{n}=5)$, S. oralis subsp. dentisani (frequently abbreviated as "S. dentisani") $(\mathrm{n}=$ 5), S. oralis subsp. oralis (frequently abbreviated as "S. oralis") $(\mathrm{n}=5), S$. oralis subsp. tigurinus (frequently abbreviated as " $S$. tigurinus") $(\mathrm{n}=5), S$. parasanguinis $(\mathrm{n}=5), S$. pneumoniae $(\mathrm{n}=$ 5), S. pyogenes $(\mathrm{n}=5)$, S. sanguinis $(\mathrm{n}=5)$, and $S$. sobrinus $(\mathrm{n}=$ 5). S. downei and S. peroris were also included but had only two and one publicly available genome, respectively. As the salivarius group streptococci were the principal target in this study, $S$. salivarius, $S$. thermophilus, and $S$. vestibularis were represented by 15,15 and 7 genomes (summing up to 37 in total), respectively. All de novo assembled genomes were annotated using Prokka pipeline version 1.13.0 (Seemann, 2014). Subsequently, coaE genes were localized and extracted from entire genomes GenBank (.gbk) files through Artemis version 18.0.3 (Carver et al., 2005). Based on coaE gene sequence alignments from the in total 154 investigated genomes, we targeted suitable conserved regions as primer binding sites specific for salivarius group streptococci. Primer sequences were designed and optimized using Primer3Plus (Untergasser et al., 2012). For the salivarius group streptococci, forward primer coaE-SG-fwd 5' $5^{\prime}$ TTAACAGGWGGYATTGCTTCAG$3^{\prime}$ and reverse primer coaE-SG-rev 5'-CTTMACCTTCT YCTTCAAATCATC-3' were designed. PCR and Sanger sequencing were performed as described previously (Conrads et al., 2017) with the following conditions: an initial denaturation step at $94^{\circ} \mathrm{C}$ for $1 \mathrm{~min}$, followed by 30 cycles (each cycle consisting of $94^{\circ} \mathrm{C}$ for $30 \mathrm{~s}, 51^{\circ} \mathrm{C}$ for $30 \mathrm{~s}$ and $72^{\circ} \mathrm{C}$ for $1 \mathrm{~min}$ ) terminated by a final extension step at $72^{\circ} \mathrm{C}$ for $4 \mathrm{~min}$. The primer specificity was carefully ascertained in silico; however, one $S$. mitis isolate (OMI-317) was included as a negative control to proof salivarius group primer specificity in vitro for every run. PCR products purity and length were confirmed by conventional agarose gel electrophoresis.

\section{Molecular Analysis of Isolates}

To evaluate the use of coaE as molecular typing target, we included a collection of 30 salivarius group strains (S. vestibularis, $\mathrm{n}=8$; $S$. thermophilus $\mathrm{n}=9$, and $S$. salivarius $\mathrm{n}=13$, labelled "OMI" as from the Division of Oral Microbiology and Immunology strain collection) that were isolated from human (blood $n=15$, saliva $n=9$, feces $n=3$, hip joint puncture $n=1$, aortic valve $n=1$, clinical data 
not available $n=1$ ) (for further data see Table S1). Isolates were grown overnight on Columbia colistin-nalidixic acid (CNA) agar with 5\% (vol/vol) sheep blood (Becton Dickinson, Heidelberg, Germany) at $37^{\circ} \mathrm{C}$ with an atmosphere of $8 \% \mathrm{CO}_{2}$. All isolates were pre-identified and pre-classified using MALDI-TOF MS (Biotyper, Bruker Daltonik) according to the manufacturer. For DNA-preparation, an appropriate biomass was collected and re-

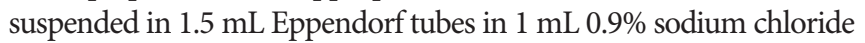
$(\mathrm{NaCl})$. Tubes were centrifuged for $1 \mathrm{~min}$ at $8,000 \mathrm{rpm}$ and the supernatant was discarded. Remained pellets were treated with a mixture of lysozyme and mutanolysin (LM) and incubated for 30 min at $37^{\circ} \mathrm{C}$ to disrupt the cell walls. Subsequently, the genomic DNA was extracted using the QIAamp ${ }^{\circledR}$ DNA Mini Kit (Qiagen, USA) according to the manufacturer's instructions.

\section{CoaE Phylogenetic Tree Reconstruction}

A Multi-Fasta file of coaE gene sequences was aligned using ClustalW algorithm implemented in MEGAX software version 10.0.5 (Kumar et al., 2018). For determining the best-fit evolutionary model of nucleotide substitutions, we used the model selection function (Find Best DNA/Protein Models) implemented in MEGAX software. A model with the lowest Bayesian Information Criterion (BIC) scores was considered to describe the substitution pattern the best, and subsequently, was used for the phylogeny reconstruction. The multiple sequence alignments were used to reconstruct a maximum likelihood (ML) phylogenetic tree with PhyML using default settings and applying complete deletion of gaps/missing data and bootstrap test of 1000 replicates. In addition to the ML method, the neighbor-joining (NJ) method with complete deletion of gaps/ missing data and bootstrap test of 1000 replicates that is implemented in MEGAX software (Kumar et al., 2018) was used to investigate the self-reliance of the phylogenetic hypothesis of salivarius group.

\section{RESULTS}

Among the salivarius group genomes, the coaE gene sequence length was conserved with 594 base pairs (bp). Using this sequence as template, we designed primers targeting the variable region of locus_tag="SSAL8618_RS03260" in the reference genome of $S$. salivarius strain NCTC 8618 (ATCC 7073) (accession number: NZ_CP009913) at nucleotide positions 13-34 and 538-561 (calculated amplicon length 549 $\mathrm{bp}$ ), respectively. A total of 30 salivarius group streptococci isolates were characterized using the $c o a E$ typing approach. This PCR revealed indeed amplicons for all salivarius group isolates, while the laboratory negative-control ( $S$. mitis isolate OMI-317) showed no amplification. Sequencing the PCR products of all isolates revealed the expected amplicon size with 549 bp in length. Subsequently, the phylogenetic tree based on the coaE gene sequence of these $30 \mathrm{OMI}$-isolates and of the 37 publicly available salivarius group genomes was constructed using ML method and Tamura 3-parameter substitution model with a discrete gamma distribution as it was shown by model testing to be the best-fit evolutionary model $(\mathrm{BIC}=6398.365)$ (Figure 1A). The ML tree showed two main distinct clusters, one representing $S$. salivarius and one consisting of $S$. thermophilus and S. vestibularis. S. vestibularis had several phylogenetically distinct sub-clusters, differing by 31 single nucleotide polymorphisms (SNPs) in average, that were clearly located closer to the $S$. thermophilus than to the $S$. salivarius clade. This phylogenetic clustering was confirmed by a separate NJ phylogenetic method (Figure 1B), which revealed identical two clusters of $S$. salivarius, $S$. thermophilus and $S$. vestibularis with high bootstrap values comparable to those obtained from the ML method (Figure 1A).

The isolates OMI357, OMI430, OMI431, OMI433, and OMI434 (marked by * in Figure $\mathbf{1}$ and Table 1) were primarily assigned as $S$. vestibularis based on MALDI-TOF MS, but showing uncertain results with a score between 2.07 to 2.23 for S. vestibularis as first-best match and between 2.04 too 2.15 for $S$. salivarius as second-best match. Clearly, coaE gene typing placed these isolates within the $S$. salivarius cluster suggesting a wrong scoring order by MALDI-TOF MS. Another example of misidentification by MALDI-TOF MS was S. salivarius strain K12 (subculture OMI428, marked by ${ }^{\S}$ in Figure 1) misidentified as $S$. vestibularis (Table 1). However, for all previously mentioned isolates, we used in Figure $\mathbf{1}$ the right identification species name (S. salivarius) according to coaE gene typing. The imported coaE sequence of strain K12 and the sequencing result of our own K12 subculture (OMI428) were identical, as expected. Taken together, our results show that MALDI-TOF MS failed to assign almost $50 \%$ of the investigated S. salivarius isolates, while all isolates belonging to $S$. thermophilus and S. vestibularis were correctly identified (Table 1).

In order to investigate the coaE gene variability among the 67 salivarius group genomes/isolates, we calculated the mean distance among the different species-specific clades. The results revealed that the $S$. salivarius clade was most divergent from both, S. vestibularis and S. thermophilus clade by 81 and 93 nucleotide differences, respectively. While $S$. vestibularis and $S$. thermophilus were more closely related and differed by 50 nucleotide substitutions only. Furthermore, we detected a diversity as low as 7 SNPs among isolates of the $S$. thermophilus clade, while the within clade distances of $S$. salivarius and $S$. vestibularis were 27 SNPs and 31 SNPs, respectively.

We elucidated the base composition and genetic variability of the complete coaE gene sequence (from ATG to Stop-codon TGA/TAA/TAG, the latter version TAG used in salivarius group), among 154 publicly available genomes that represent 23 streptococcal and four non-streptococcal species. Extracting the coaE gene sequences from these 154 genomes revealed a variable and - by excluding $S$. infantis species - specific gene length that ranged between 525 to $630 \mathrm{bp}$. For example, the largest coaE gene was detected in E. faecium (630 bp), while the shortest was detected in two out of five genomes of S. infantis (525 bp). This heterogeneity in length came along with a large variability in base composition among the five coaE gene sequences of $S$. infantis. But with this exception of S. infantis, 

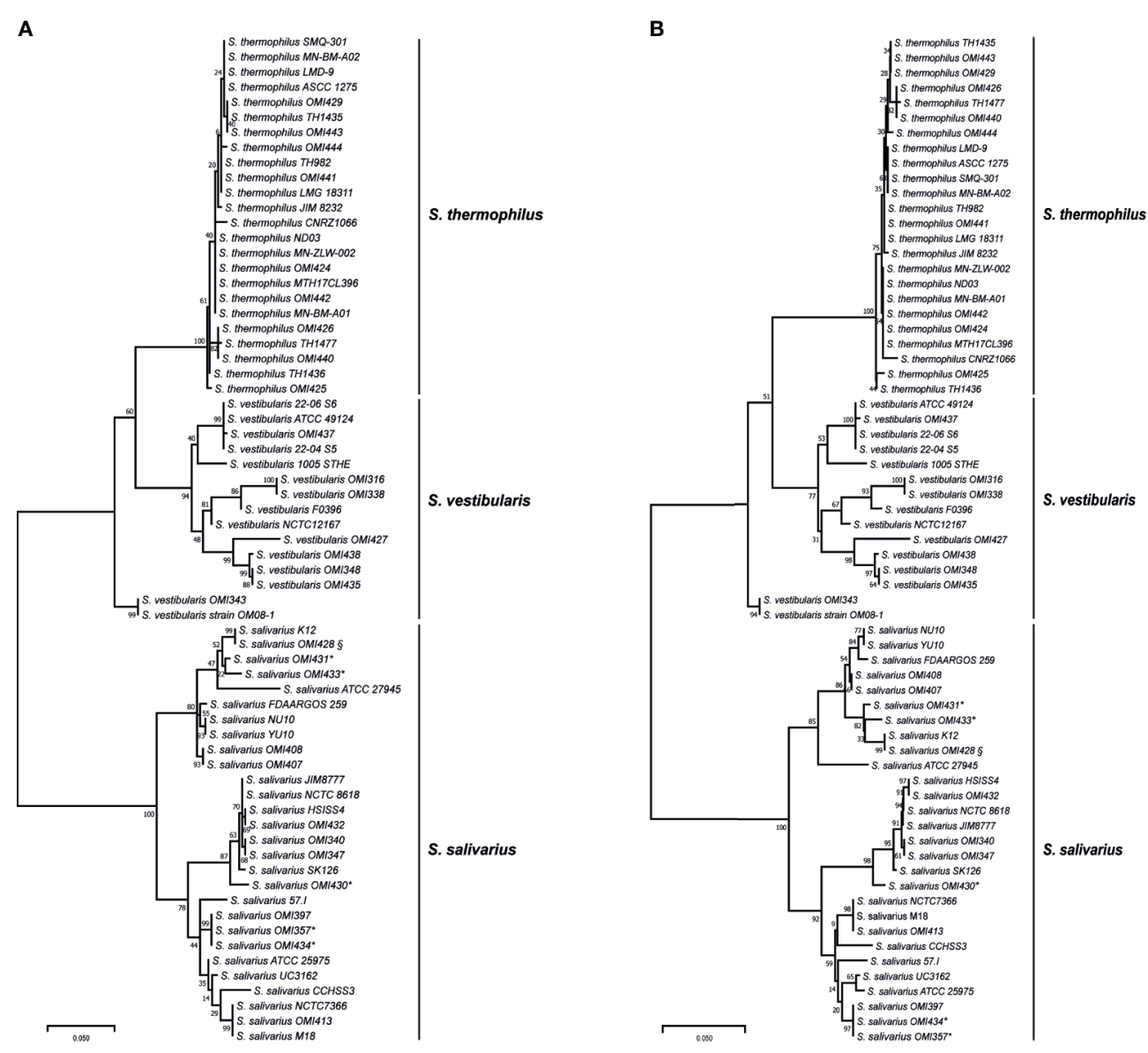

FIGURE 1 | Maximum likelihood (A) and neighbor-joining (B) phylogenetic trees based on the coaE gene sequence of 30 isolates (OMI) and from 37 publicly available salivarius group streptococci genomes imported from NCBI database. * represents isolates that were primarily assigned as "S. vestibularis" based on MALDI-TOF MS, while ${ }^{\S}$ shows a subculture of $S$. salivarius strain K12, OMI428, which was misidentified as "S. vestibularis" by MALDI-TOF MS. All these isolates were confirmed as S. salivarius by applying coaE gene typing, suggesting a wrong scoring order by MALDI-TOF MS. Tamura 3-parameter was used as substitution model with a discrete gamma distribution and bootstrap test of 1000 replicates.

probably indicating either misclassification or subspecies formation, the coaE gene had an average of $86 \%$ conserved positions on species level and heterogeneity was only found among different species.

In order to visualize the relationships among the 154 genomes investigated; we constructed a phylogenetic tree based on the coaE gene sequence alignment (Figure 2) using ML method and Tamura 3-parameter substitution model with a discrete gamma distribution of invariable sites as it was shown by model testing to be the best-fit evolutionary model $(\mathrm{BIC}=36733.568)$. Interestingly, the ML tree revealed that the 154 genomes made up two main clades with distinct phylogenetic clusters that were strongly associated with the different bacterial species. One distinct phylogenetic clade (clade 1) contained all nonstreptococcal genomes consisting of four clusters of $S$. aureus, L. monocytogenes, E. faecalis, and E. faecium. On the other hand, the 134 genomes belonging to the genus Streptococcus made up the second distinct clade (clade 2) that was divided into three main clusters. The streptococcal clade differed from the non- streptococcal clade with an average of 304 nucleotide substitutions, while the within clade diversity showed comparable number of nucleotide differences (224 for clade 1 and 222 for clade 2).

Each of the three main streptococcal clusters showed groups and species-specific, but not subspecies-specific sub-clusters (Figure 2). For instance, the salivarius group members $S$. salivarius, $S$. vestibularis and $S$. thermophilus together formed a group-specific main cluster (cluster 1) that was divided into individual species-specific subclusters. In addition, most genomes of mitis group members $(n=40)$ made up cluster 2 including $S$. australis, S. parasanguinis, S. infantis, S. mitis, S. pneumoniae, $S$. oralis subsp. tigurinus, $S$. oralis subsp. dentisani, $S$. oralis subsp. oralis, and $S$. peroris, the latter represented by only one publicly available genome. Similar to cluster 1 , speciesspecific subclusters were also demonstrated in cluster 2 . Furthermore, genomes of S. oralis subsp. tigurinus, S. oralis subsp. dentisani and $S$. oralis subsp. oralis $(n=15)$ clustered together with limited species-specific resolution, especially for 
TABLE 1 | Comparison between MALDI-TOF MS and coaE typing results of the 30 salivarius group streptococcal (OMI) isolates included in this study.

\begin{tabular}{|c|c|c|c|c|c|}
\hline Isolate & First match & Firstmatch score & Second match & Second match score & coaE identification \\
\hline OMI340 & $S$ & 2.33 & $V$ & 2.26 & $S$ \\
\hline OMI347 & $S$ & 2.31 & $S$ & 2.25 & $S$ \\
\hline OMI397 & $S$ & 2.22 & $V$ & 2.21 & $S$ \\
\hline OMI407 & $S$ & 2.27 & $S$ & 2.12 & $S$ \\
\hline OMI408 & $S$ & 2.24 & $S$ & 2.22 & $S$ \\
\hline OMI413 & $S$ & 2.10 & $S$ & 2.09 & $S$ \\
\hline OMI428§ & V & 2.18 & V & 2.04 & $S$ \\
\hline OMI430* & V & 2.16 & $S$ & 2.13 & $S$ \\
\hline OMI431* & V & 2.13 & $S$ & 2.09 & $S$ \\
\hline OMI432 & $S$ & 2.28 & $S$ & 2.10 & $S$ \\
\hline OMI433* & V & 2.16 & $S$ & 2.06 & $S$ \\
\hline OMI434* & $V$ & 2.07 & $S$ & 2.04 & $S$ \\
\hline OMI357* & V & 2.23 & $S$ & 2.15 & $S$ \\
\hline OMI424 & $T$ & 2.29 & $T$ & 1.93 & $T$ \\
\hline OMI425 & $T$ & 2.25 & $T$ & 2.13 & $T$ \\
\hline OMI426 & $T$ & 2.08 & $T$ & 2.01 & $T$ \\
\hline OMI429 & $T$ & 1.91 & $T$ & 1.90 & $T$ \\
\hline OMI440 & $T$ & 2.07 & $T$ & 1.96 & $T$ \\
\hline OMI441 & $T$ & 2.22 & $T$ & 2.11 & $T$ \\
\hline OMI442 & $T$ & 2.06 & $T$ & 1.88 & $T$ \\
\hline OMI443 & $T$ & 2.02 & $T$ & 1.99 & $T$ \\
\hline OMI444 & $T$ & 2.01 & $T$ & 1.94 & $T$ \\
\hline OMI316 & $V$ & 2.49 & V & 2.40 & $V$ \\
\hline OMI338 & $V$ & 2.33 & $V$ & 2.32 & $V$ \\
\hline ОМІ343 & V & 2.42 & $V$ & 2.36 & V \\
\hline OMI348 & $V$ & 2.00 & $V$ & 1.98 & $V$ \\
\hline OMI427 & V & 1.91 & V & 1.90 & V \\
\hline OMI435 & $V$ & 1.96 & $V$ & 1.86 & $V$ \\
\hline OMI437 & V & 2.41 & $V$ & 2.28 & V \\
\hline OMI438 & $V$ & 2.02 & $V$ & 1.92 & $V$ \\
\hline
\end{tabular}

S, S. salivarius; V, S. vestibularis; and T, S. thermophilus.

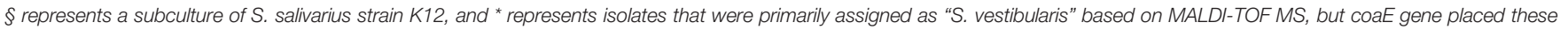
isolates in the S. salivarius cluster.

S. oralis genomes. For instance, two mixed subclusters (S. oralis) S. tigurinus) included genomes of $S$. oralis subsp. tigurinus and $S$. oralis subsp. oralis. In addition, S. oralis subsp. dentisani made up a distinct subcluster of four genomes, by excluding strain CECT7746, which is an important probiotic that clustered among $S$. oralis subsp. oralis and $S$. oralis subsp. tigurinus genomes (Figure 2). Within cluster 2, S. mitis and $S$. pneumoniae were the most closely related species with 44 nucleotide differences only, while the highest number of substitutions $(\mathrm{n}=214)$ was detected between $S$. parasanguinis and S. peroris (Figure 3).

Cluster 3 harbored the highest number of the investigated streptococcal species $(n=12)$ that represented four different streptococcal groups (pyogenic, bovis, mutans, anginosus and mitis). Interestingly, the remaining members of mitis group (S. sanguinis, S. gordonii, and S. cristatus), that were included in this analysis, were located in cluster 3 and differed from mitis members in cluster 2 by at least 195 nucleotide substitutions for $S$. australis and S. cristatus. The pyogenic group, represented by S. pyogenes and S. agalactiae did not directly cluster together but were separated by $S$. equinus from the bovis group. However, the pairwise sequence analysis revealed that coaE sequences of S. pyogenes and S. agalactiae differed by 184 nucleotides, while S. equinus had 186 and 187 nucleotide differences to S. agalactiae and $S$. pyogenes, respectively. In cluster 3, we detected the lowest number of nucleotide substitutions $(\mathrm{n}=25)$ among $S$. sobrinus and $S$. downei, while $S$. cristatus and $S$. pyogenes were the most divergent by 213 nucleotides (Figure 3).

\section{DISCUSSION}

Several studies have reported the identification and typing challenges of viridans group streptococci. For instance, the use of MALDI-TOF MS revealed several limitations in the identification of certain species of streptococci such as those within the salivarius group (Murray, 2010). Similarly, the use of DNA-based identification approaches targeting different genes such as $16 \mathrm{~S}$ rRNA, groESL, rpoB, tuf, and $\operatorname{sod} A$ were unable to deliver a high discriminatory power for distinguishing between salivarius group species (Simmon et al., 2008; Angeletti et al., 2015).

In this study, we have developed a new typing approach based on the amplification and sequence analysis of coaE gene as target marker. In addition, we evaluated the use of $c o a E$ gene sequences in identifying members of salivarius group streptococci on species level. The clear distinction between salivarius group species is generally difficult, since the $16 \mathrm{~S}$ rRNA gene of all three species is almost identical (> 99\%) (Thompson et al., 2013). Furthermore, a previous study used the $t k t$ gene (encoding a 


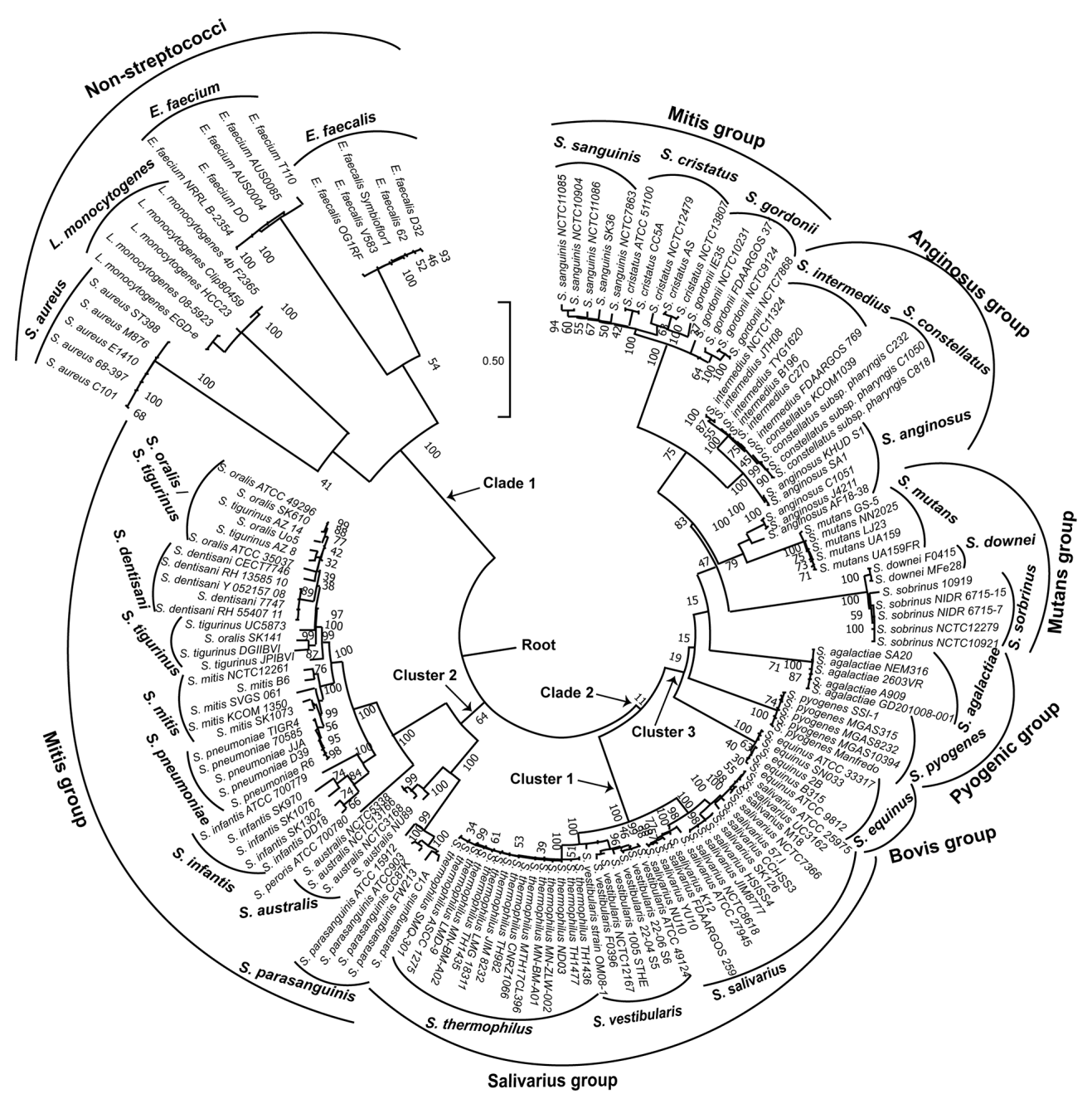

FIGURE 2 | Maximum likelihood phylogenetic tree based on the coaE gene sequence of 154 bacterial genomes (134 streptococcal and 20 non-streptococcal genomes). The tree was rooted using the non-streptococcal clade (clade 1) as an outgroup. Tamura 3-parameter was used as substitution model with a discrete gamma distribution of invariable sites and bootstrap test of 1000 replicates.

transketolase) for the differentiation between S. salivarius and S. vestibularis (Van den Bogert et al., 2013). However, it was shown that this gene exists in two completely different and variable forms as it can be acquired through horizontal gene transfer (implicating a risk of gene loss or homologous recombination), which makes it unsuitable as typing gene (Delorme et al., 2007). Another molecular typing method based on sequence analysis of the tuf gene (encoding the elongation factor $\mathrm{Tu}$ ) revealed that the salivarius group was monophyletic and $S$. salivarius compared to $S$. vestibularis isolates were $99.1 \%$ identical within a tuf sequence of 761-bp length (Picard et al., 2004).

Our results revealed that coaE gene typing divided the investigated salivarius group genomes into three speciesspecific clades and was able to identify and differentiate them precisely. Interestingly, MALDI-TOF MS typing of six $S$. salivarius isolates revealed misleading results assigning these isolates as $S$. vestibularis or S. salivarius with similar scores.
These findings are in agreement with a previous study, which showed that the identification of $S$. vestibularis using MALDITOF MS is not reliable (Angeletti et al., 2015). As a clear progress for classification of streptococci, here, coaE typing revealed a precise identification of all investigated $S$. salivarius isolates.

However, in contrast to $S$. salivarius and $S$. thermophilus isolates, we detected large coaE sequence diversity among the S. vestibularis isolates (up to 31 SNPs). In addition, we found that $S$. vestibularis and $S$. thermophilus were closer related to each other (50 nucleotide substitutions) compared to S. salivarius (81 and 93 nucleotide substitutions, respectively).

An explanation for the low diversity of 7 SNPs detected within S. thermophilus is probably that most S. thermophilus public genomes $(n=15)$ included here represent strains isolated from dairy products. As only a few S. thermophilus strains are used as starter culture for the industrial yogurt production and as their identity must be controlled and guaranteed, a low diversity is the consequence (Linares et al., 2016). However, the remaining 


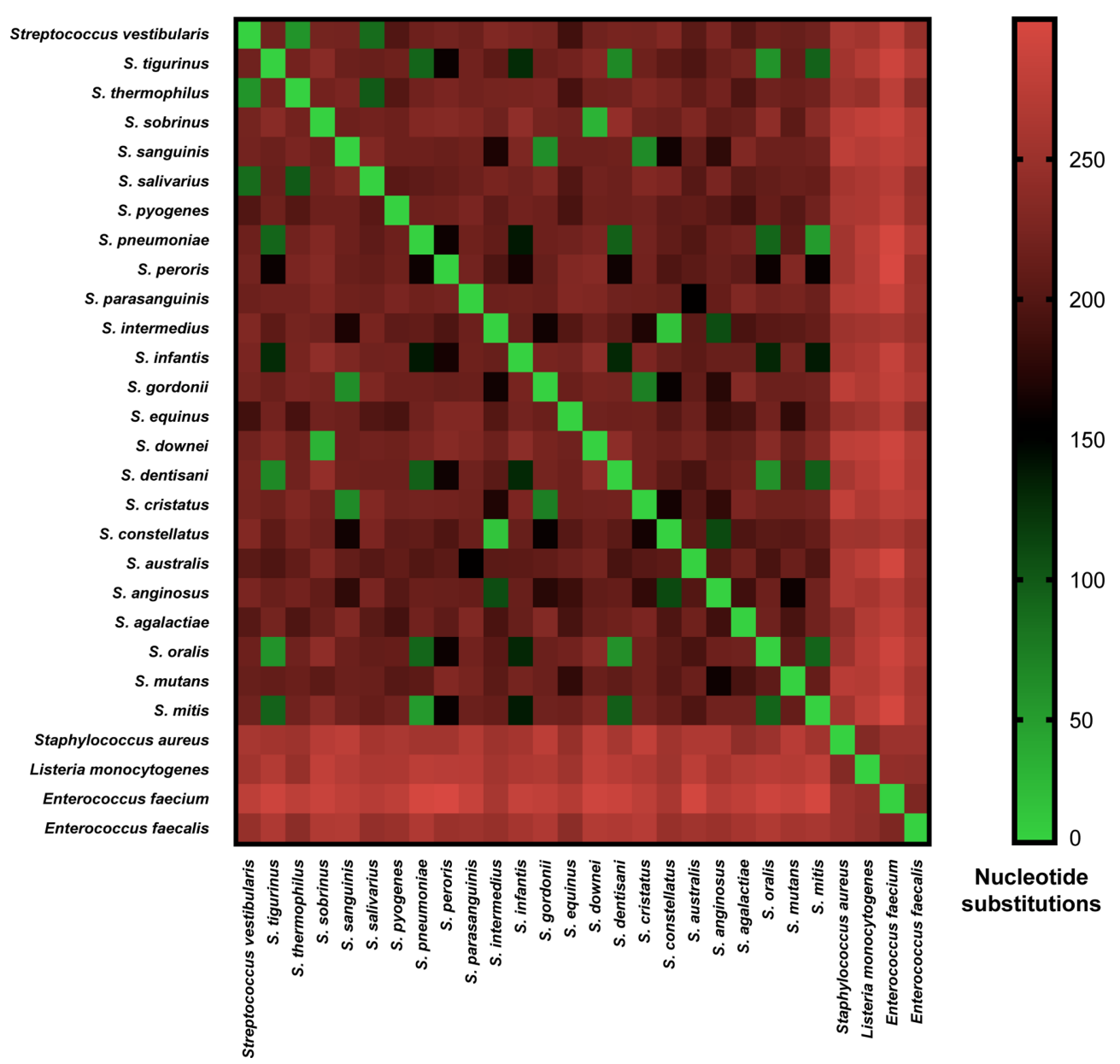

FIGURE 3 | Pairwise nucleotide substitutions heat map. Number of nucleotide substitutions over a 525-630 bp coaE gene sequence between 28 different bacterial species as a measure of evolutionary divergence.

9 isolates within S. thermophilus clade were retrieved from blood samples, suggesting close genetic relatedness among commensal and pathogenic strains. This implies that the use of further typing methods with higher discriminatory power, such as WGS, should be applied to gain a better understanding of the evolution of $S$. thermophilus pathogenic strains.

The coaE typing distinguished between streptococci and nonstreptococci species by a mean distance of 304 nucleotide differences, which emphasizes the inter-genus variation of the coaE gene. These results show that $c o a E$ is a suitable target for identifying bacterial species that belong to different genera.

However, the coaE gene typing method had a limited resolution to split the $S$. oralis subspecies genomes within the mitis group streptococci. A previous study based on the use of WGS revealed that the three $S$. oralis subspecies form subclusters - within an otherwise coherent phylogenetic clade - with relatively poor separation (Jensen et al., 2016). A former study of our own group subjecting subsp. S. oralis and S. tigurinus concluded that a clear separation between these subspecies will never be sharp (Conrads et al., 2017) and that linker or hybrid strains do exist. Such hybrids have been described in the mitis group before by Kilian et al. (2008). Taken together, neither coaE nor WGS can resolve the principal problem of hybrids.

Previous studies showed that species identification of mitis group streptococci is challenging, with exception of S. pneumoniae (Jensen et al., 2016; Velsko et al., 2019). The use of MALDI Biotyper and MALDI-TOF MS revealed misidentifications of non-S. pneumoniae mitis strains and some of them were identified as " $S$. pneumoniae" (Kärpänoja et al., 2014; Yahiaoui et al., 2019). The coaE typing might help to overcome these false "S. pneumoniae" identifications. Our phylogenetic analyses, based on coaE gene sequences revealed that the mitis group is made up of different clusters with substantial genetic variety among individual species. These findings are in agreement with a previous study based on WGS suggesting that the mitis group consists of a mixture of genetically distinct and coherent phylogenetic clades (Jensen et al., 2016).

Taken together, the sequence comparisons of coaE gene revealed its conservation across the streptococci and non-streptococci species, 
which directly supports a common ancestor origin of coaE followed by speciation of the different bacterial species. In addition, the coaE based typing approach evaluated here demonstrated its usefulness for species identification as a standalone method or as an extension of existing concatenated gene sequences for MLST. Furthermore, this method proved to be a precise (100\% correct identification of $S$. salivarius isolates) and cost-efficient (single gene) typing strategy for the investigation of salivarius group isolates. This is especially important as members of this group are frequently used as probiotics but can also cause serious clinical conditions, including blood stream infections.

In conclusion, we showed the high value of a coaE based typing approach for the precise identification of salivarius group species. The only alternative with a similar or even better discriminatory power is WGS but which is more expensive ( $\sim 200$ Euros per isolate) and time consuming ( $24-72 \mathrm{~h}$, plus upstream culture $24-96 \mathrm{~h}$ and 2 - $4 \mathrm{~h}$ downstream data analysis) and requires bioinformatics expertise accessible for only a few laboratories (Deurenberg et al., 2017; Vourli et al., 2021). Therefore, coaE analysis is fast and cost-effective as a first-line typing tool for salivarius group streptococci.

\section{DATA AVAILABILITY STATEMENT}

The datasets presented in this study can be found in online repositories. The names of the repository/repositories and accession number(s) can be found below: https://www.ddbj.nig. ac.jp/, accession numbers LC621196 to LC621225.

\section{ETHICS STATEMENT}

The oral and fecal S. salivarius and S. vestibularis isolates $(\mathrm{n}=11)$ were obtained from the study velphoro and impact on the oral cavity and gut microbiome. This study was approved by the Ethics Committee (EK No. 270/17) of the Medical Faculty of RWTH

\section{REFERENCES}

Angeletti, S., Dicuonzo, G., Avola, A., Crea, F., Dedej, E., Vailati, F., et al. (2015). Viridans Group Streptococci Clinical Isolates: MALDI-TOF Mass Spectrometry Versus Gene Sequence-Based Identification. PloS One 10, e0120502. doi: 10.1371/journal.pone.0120502

Ballinger, E., Mosior, J., Hartman, T., Burns-Huang, K., Gold, B., Morris, R., et al. (2019). Opposing Reactions in Coenzyme A Metabolism Sensitize Mycobacterium Tuberculosis to Enzyme Inhibition. Science 363, eaau8959. doi: $10.1126 /$ science.aau8959

Carver, T. J., Rutherford, K. M., Berriman, M., Rajandream, M.-A., Barrell, B. G., and Parkhill, J. (2005). ACT: The Artemis Comparison Tool. Bioinformatics 21, 3422-3423. doi: 10.1093/bioinformatics/bti553

Conrads, G., Barth, S., Möckel, M., Lenz, L., van der Linden, M., and Henne, K. (2017). Streptococcus Tigurinus Is Frequent Among gtfR-Negative Streptococcus Oralis Isolates and in the Human Oral Cavity, But Highly Virulent Strains are Uncommon. J. Oral. Microbiol. 9, 1307079. doi: 10.1080/ 20002297.2017.1307079

Corredoira, J. C., Alonso, M. P., García, J. F., Casariego, E., Coira, A., Rodriguez, A., et al. (2005). Clinical Characteristics and Significance of Streptococcus Salivarius Bacteremia and Streptococcus Bovis Bacteremia: A Prospective 16-
Aachen University. All remaining 19 isolates including seven $S$. salivarius, three $S$. vestibularis and all nine $S$. thermophilus were received from the German National Reference Center for Streptococci. For these 19 isolates an ethical approval or patients' consent was not required since these isolates resulted from routine microbiological diagnostic procedures as requested by the treating physician. All isolates were anonymized and only sample type (saliva, feces or blood) was registered if available.

\section{AUTHOR CONTRIBUTIONS}

MA comprehended the idea and wrote the manuscript. MA and GC designed the study. GW performed the experiments. GW and MA analysed the data. All authors contributed to the article and approved the submitted version.

\section{FUNDING}

The study was funded by the START Program of the RWTH Aachen University Hospital (STREPTORANTES \#109/19).

\section{ACKNOWLEDGMENTS}

We thank Mark van der Linden (National Reference Center for Streptococci, University Hospital Aachen, Germany) for providing streptococcal isolates. We are grateful to Mrs. Beate Melzer-Krick for her excellent technical assistance.

\section{SUPPLEMENTARY MATERIAL}

The Supplementary Material for this article can be found online at: https://www.frontiersin.org/articles/10.3389/fcimb.2021. 685657/full\#supplementary-material

Year Study. Eur. J. Clin. Microbiol. Infect. Dis. 24, 250-255. doi: 10.1007/ s10096-005-1314-x

Delorme, C., Poyart, C., Ehrlich, S. D., and Renault, P. (2007). Extent of Horizontal Gene Transfer in Evolution of Streptococci of the Salivarius Group. J. Bacteriol. 189, 1330-1341. doi: 10.1128/JB.01058-06

Deurenberg, R. H., Bathoorn, E., Chlebowicz, M. A., Couto, N., Ferdous, M. García-Cobos, S., et al. (2017). Application of Next Generation Sequencing in Clinical Microbiology and Infection Prevention. J. Biotechnol. 243, 16-24. doi: 10.1016/j.jbiotec.2016.12.022

Friedrichs, C., Rodloff, A. C., Chhatwal, G. S., Schellenberger, W., and Eschrich, K. (2007). Rapid Identification of Viridans Streptococci by Mass Spectrometric Discrimination. J. Clin. Microbiol. 45, 2392-2397. doi: 10.1128/JCM.00556-07 Galperin, M. Y., Walker, D. R., and Koonin, E. V. (1998). Analogous Enzymes: Independent Inventions in Enzyme Evolution. Genome Res. 8, 779-790. doi: $10.1101 /$ gr.8.8.779

Jensen, A., Scholz, C. F. P., and Kilian, M. (2016). Re-Evaluation of the Taxonomy of the Mitis Group of the Genus Streptococcus Based on Whole Genome Phylogenetic Analyses, and Proposed Reclassification of Streptococcus Dentisani as Streptococcus Oralis Subsp. Dentisani Comb. Nov., Streptococcus Tigurinus as Streptococcus Oralis Subsp. Tigurinus Comb. Nov., and Streptococcus Oligofermentans as a Later Synonym of 
Streptococcus Cristatus. Int. J. Syst. Evol. Microbiol. 66, 4803-4820. doi: 10.1099/ijsem.0.001433

Kärpänoja, P., Harju, I., Rantakokko-Jalava, K., Haanperä, M., and Sarkkinen, H. (2014). Evaluation of Two Matrix-Assisted Laser Desorption Ionization-Time of Flight Mass Spectrometry Systems for Identification of Viridans Group Streptococci. Eur. J. Clin. Microbiol. Infect. Dis. 33, 779-788. doi: 10.1007/s10096-013-2012-8

Kilian, M., Poulsen, K., Blomqvist, T., Håvarstein, L. S., Bek-Thomsen, M., Tettelin, H., et al. (2008). Evolution of Streptococcus Pneumoniae and Its Close Commensal Relatives. PloS One 3, e2683. doi: 10.1371/journal.pone.0002683

Kumar, S., Stecher, G., Li, M., Knyaz, C., and Tamura, K. (2018). MEGA X: Molecular Evolutionary Genetics Analysis Across Computing Platforms. Mol. Biol. Evol. 35, 1547-1549. doi: 10.1093/molbev/msy096

Lartigue, M.-F., Hery-Arnaud, G., Haguenoer, E., Domelier, A.-S., Schmit, P.-O., van der Mee-Marquet, N., et al. (2009). Identification of Streptococcus Agalactiae Isolates From Various Phylogenetic Lineages by Matrix-Assisted Laser Desorption Ionization-Time of Flight Mass Spectrometry. J. Clin. Microbiol. 47, 2284-2287. doi: 10.1128/JCM.00175-09

Linares, D. M., O'Callaghan, T. F., O'Connor, P. M., Ross, R. P., and Stanton, C. (2016). Streptococcus Thermophilus APC151 Strain Is Suitable for the Manufacture of Naturally GABA-Enriched Bioactive Yogurt. Front. Microbiol. 7, 1876. doi: 10.3389/fmicb.2016.01876

Mishra, P. K., Park, P. K., and Drueckhammer, D. G. (2001). Identification of yacE (coaE) as the Structural Gene for Dephosphocoenzyme A Kinase in Escherichia Coli K-12. J. Bacteriol. 183, 2774-2778. doi: 10.1128/ JB.183.9.2774-2778.2001

Murray, P. R. (2010). Matrix-Assisted Laser Desorption Ionization Time-of-Flight Mass Spectrometry: Usefulness for Taxonomy and Epidemiology. Clin. Microbiol. Infect. 16, 1626-1630. doi: 10.1111/j.1469-0691.2010.03364.x

Omelchenko, M. V., Galperin, M. Y., Wolf, Y. I., and Koonin, E. V. (2010). NonHomologous Isofunctional Enzymes: A Systematic Analysis of Alternative Solutions in Enzyme Evolution. Biol. Direct 5, 31. doi: 10.1186/1745-6150-5-31

Picard, F. J., Ke, D., Boudreau, D. K., Boissinot, M., Huletsky, A., Richard, D., et al. (2004). Use of Tuf Sequences for Genus-Specific PCR Detection and Phylogenetic Analysis of 28 Streptococcal Species. J. Clin. Microbiol. 42, 3686-3695. doi: 10.1128/JCM.42.8.3686-3695.2004

Seemann, T. (2014). Prokka: Rapid Prokaryotic Genome Annotation. Bioinformatics Oxf. Engl. 30, 2068-2069. doi: 10.1093/bioinformatics/btu153

Shewmaker, P. L., Gertz, R. E., Kim, C. Y., de Fijter, S., DiOrio, M., Moore, M. R., et al. (2010). Streptococcus Salivarius Meningitis Case Strain Traced to Oral Flora of Anesthesiologist. J. Clin. Microbiol. 48, 2589-2591. doi: 10.1128/JCM.00426-10

Shimosaka, T., Makarova, K. S., Koonin, E. V., and Atomi, H. (2019). Identification of Dephospho-Coenzyme A (Dephospho-CoA) Kinase in Thermococcus Kodakarensis and Elucidation of the Entire CoA Biosynthesis Pathway in Archaea. mBio 10, e01146-e01119. doi: 10.1128/mBio.01146-19. mbio/10/4/mBio.01146-19.atom.

Simmon, K. E., Hall, L., Woods, C. W., Marco, F., Miro, J. M., Cabell, C., et al (2008). Phylogenetic Analysis of Viridans Group Streptococci Causing Endocarditis. J. Clin. Microbiol. 46, 3087-3090. doi: 10.1128/JCM.00920-08

Spry, C., Kirk, K., and Saliba, K. J. (2008). Coenzyme A Biosynthesis: An Antimicrobial Drug Target. FEMS Microbiol. Rev. 32, 56-106. doi: 10.1111/ j.1574-6976.2007.00093.x
Srinivasan, V., Gertz, R. E.Jr., Shewmaker, P. L., Patrick, S., Chitnis, A. S., O'Connell, H., et al. (2012). Using PCR-Based Detection and Genotyping to Trace Streptococcus Salivarius Meningitis Outbreak Strain to Oral Flora of Radiology Physician Assistant. PloS One 7, e32169. doi: 10.1371/ journal.pone.0032169

Thompson, C. C., Emmel, V. E., Fonseca, E. L., Marin, M. A., and Vicente, A. C. P. (2013). Streptococcal Taxonomy Based on Genome Sequence Analyses. F1000Research 2, 67. doi: 10.12688/f1000research.2-67.v1

Untergasser, A., Cutcutache, I., Koressaar, T., Ye, J., Faircloth, B. C., Remm, M., et al. (2012). Primer3-New Capabilities and Interfaces. Nucleic Acids Res. 40, e115-e115. doi: 10.1093/nar/gks596

Van den Bogert, B., Boekhorst, J., Herrmann, R., Smid, E. J., Zoetendal, E. G., and Kleerebezem, M. (2013). Comparative Genomics Analysis of Streptococcus Isolates From the Human Small Intestine Reveals Their Adaptation to a Highly Dynamic Ecosystem. PloS One 8, e83418. doi: 10.1371/journal.pone.0083418

Velsko, I. M., Perez, M. S., and Richards, V. P. (2019). Resolving Phylogenetic Relationships for Streptococcus Mitis and Streptococcus Oralis Through Coreand Pan-Genome Analyses. Genome Biol. Evol. 11, 1077-1087. doi: 10.1093/ gbe/evz049

Vourli, S., Kontos, F., and Pournaras, S. (2021). "WGS for Bacterial Identification and Susceptibility Testing in the Clinical Lab", in Application and Integration of Omics-Powered Diagnostics in Clinical and Public Health Microbiology. Eds. J. Moran-Gilad and Y. Yagel (Cham: Springer International Publishing), 2544. doi: 10.1007/978-3-030-62155-1_3

Yahiaoui, R. Y., Goessens, W. H., Stobberingh, E. E., and Verbon, A. (2020). Differentiation Between Streptococcus Pneumoniae and Other Viridans Group Streptococci by Matrix-Assisted Laser Desorption/Ionization-Time of Flight Mass Spectrometry (MALDI-TOF Ms). Clin. Microbiol. Infect. 26, S1198743X19306263. doi: 10.1016/j.cmi.2019.11.024

Yu, J., Sun, Z., Liu, W., Xi, X., Song, Y., Xu, H., et al. (2015). Multilocus Sequence Typing of Streptococcus Thermophilus From Naturally Fermented Dairy Foods in China and Mongolia. BMC Microbiol. 15, 236. doi: 10.1186/s12866015-0551-0

Conflict of Interest: The authors declare that the research was conducted in the absence of any commercial or financial relationships that could be construed as a potential conflict of interest.

Publisher's Note: All claims expressed in this article are solely those of the authors and do not necessarily represent those of their affiliated organizations, or those of the publisher, the editors and the reviewers. Any product that may be evaluated in this article, or claim that may be made by its manufacturer, is not guaranteed or endorsed by the publisher.

Copyright (c) 2021 Abdelbary, Wilms and Conrads. This is an open-access article distributed under the terms of the Creative Commons Attribution License (CC BY). The use, distribution or reproduction in other forums is permitted, provided the original author(s) and the copyright owner(s) are credited and that the original publication in this journal is cited, in accordance with accepted academic practice. No use, distribution or reproduction is permitted which does not comply with these terms. 